\title{
Use of Ovulation Predictor Kits as Adjuncts When Using Fertility Awareness Methods (FAMs): A Pilot Study
}

\author{
Rene Leiva, MD, CM, CCFP, Ula Burhan, MSc, Edmond Kyrillos, MD, CCFP, \\ Richard Febring, PhD, Robin McLaren, PhD, Catherine Dalzell, PhD, \\ and Elizabeth Tanguay, BSc
}

Purpose: Difficult clinical signs such as confusing cervical mucus or erratic basal body temperature can make the use of fertility awareness methods (FAMs) difficult in some cases. The goal of this study was to assess the feasibility of using a cheap urinary luteinizing hormone (LH)-surge identification kit as an adjunct to identify the infertile phase after ovulation when facing these scenarios.

Methods: The study used a block-allocation, crossover, 2-arm methodology (LH kit/FAM vs FAM only). Comparison of the 2 arms was done with regard to the accuracy of identification (yes/no) of the luteal phase in each cycle as confirmed by serum progesterone concentrations.

Results: We recruited 23 Canadian women currently using FAM, aged 18 to 48 years, who have had menstrual cycles 25 to 35 days long for the past 3 months and perceive themselves to have difficulty with identifying the infertile phase after ovulation. LH kits identified $100 \%$ of the luteal phases, whereas FAM indentified 87\% (statistically significant). In those identified cycles, LH kits provided a mean of 10.3 days of infertility, and FAM only provided 10 days of infertility (not statistically significant).

Conclusions: Among this population, LH kits may offer an adjunct for women who may wish to have an additional double-check. However, there are still clinical circumstances when even an LH kit does not provide confirmation. More research in this area is encouraged. (J Am Board Fam Med 2014;27: 427-429.)

Keywords: Fertility, Gynecology, Luteinizing Hormone, Natural Family Planning, Progesterone

Fertility awareness methods (FAMs) are effective methods of family planning that make use of clinical signs to monitor female fertility. ${ }^{1}$ They can provide a valid option for women for whom other methods are not desirable because of medical con-

This article was externally peer reviewed.

Submitted 10 September 2013; revised 3 January 2014; accepted 13 January 2014.

From the Bruyère Research Institute, CT Lamont Primary Health Care Research Centre, Ottawa, Ontario, Canada (RL; RM; CD); the Department of Family Medicine (RL, EK), the Faculty of Medicine (UB), and the Faculty of Nursing (ET), University of Ottawa, Ottawa, Ontario, Canada; and the College of Nursing (RF), Marquette University, Milwaukee, WI.

Funding: Support was provided by the Department of Family Medicine of the University of Ottawa through its C.T. Lamont Primary Health Care Research Centre.

Conflict of interest: none declared.

Corresponding author: Rene Leiva, MD, CM, CCFP, Department of Family Medicine, University of Ottawa, 43 Bruyère St., Ottawa, Ontario, K1N 5C8 Canada (E-mail: rene.leiva@mail.mcgill.ca). traindications or environmental, philosophical, or religious conflicts.

However, difficult signs such as confusing cervical mucus or erratic basal body temperature make the use of these methods difficult in some cases. Thus new technological approaches are sought to make FAMs easier, more effective, and cheaper. ${ }^{2}$ The purpose of this study was to assess the feasibility of using a urinary luteinizing hormone (LH)surge identification kit (sensitivity, $20 \mathrm{mIU} / \mathrm{mL}$; $\$ 1.00 /$ kit; BTNX Inc., Toronto, ON, Canada) as an adjunct to identify the infertile phase after ovulation when facing challenging scenarios.

\section{Methods}

The study consisted of a block allocation, crossover, 2-arm methodology (Figure 1). We allocated half of the participants to use the LH kit concurrently with a FAM during a menstrual cycle (arm 
Figure 1. Participants' group allocation. Arm 1 included those using the luteinizing hormone kit and the fertility awareness method (FAM), whereas arm 2 included those using only FAMs.

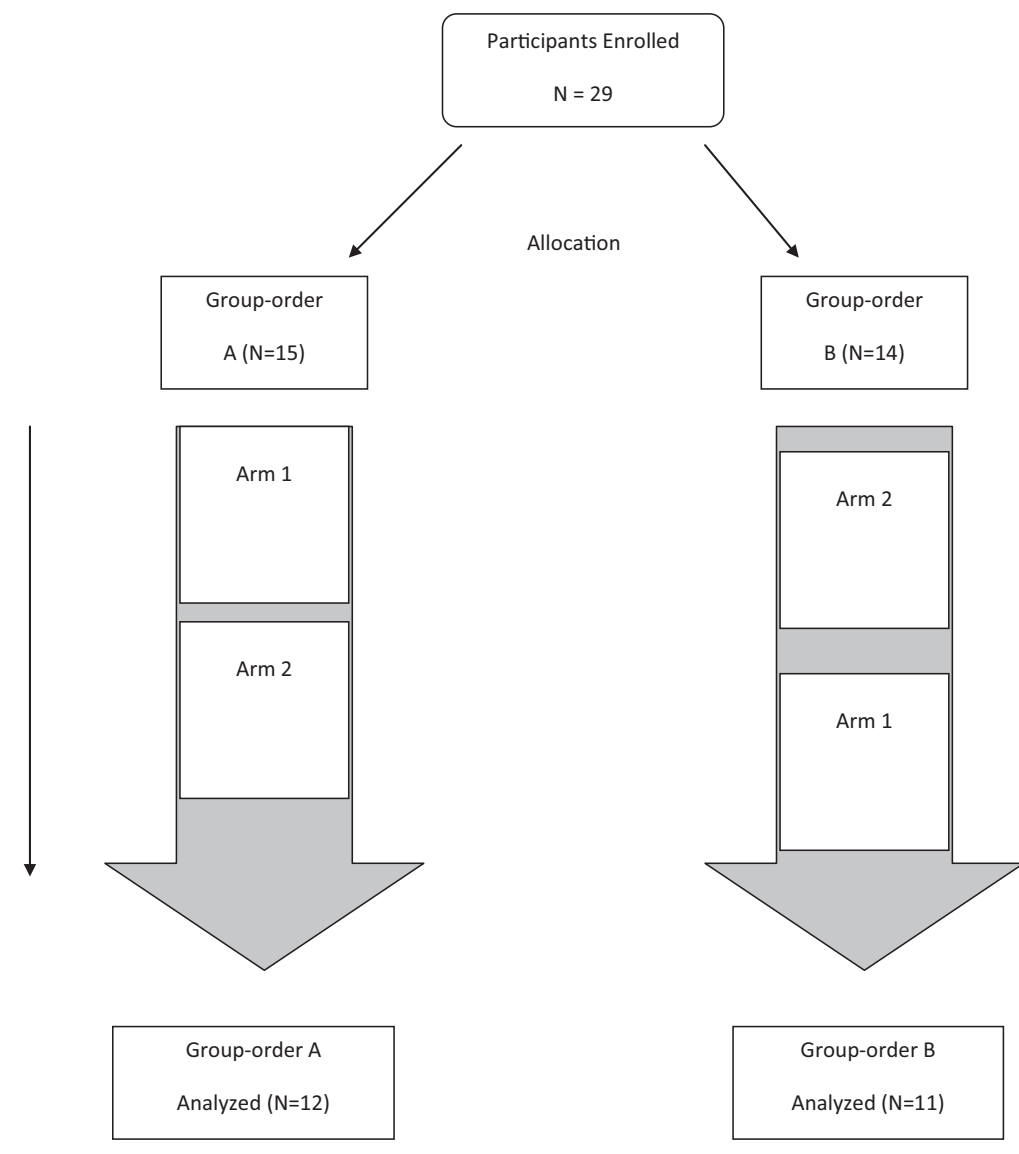

1); subsequently, a cycle with a FAM-only method was used (arm 2) (group A). The other half of participants started with opposite sequence (group B). Participants were allocated to one of the groups depending on their order of enrollment into the study. The main investigator (RL) flipped a coin to assign the first participant to a specific group, and the next participant was assigned to the alternate group, and so on. Allocation assignment occurred in similar pattern for each participant until enrolment completed. This method was used to account for a potential learning effect since each participant was exposed to the 2 arms. Ethical approval for this study was granted by the ethics and research boards of the Ottawa Hospital and Bruyere Continuing Care Hospital in Ottawa, Canada.

Each woman started once-a-day testing on the sixth day following her first day of menses until she developed a surge in LH (ie, a positive result per test's instructions) and continuing until she received negative results for 3 consecutive days. On the third day following the last day of a positive urine LH test, a serum progesterone test was done to confirm presence of the luteal phase (serum progesterone concentration $>10 \mathrm{nmol} / \mathrm{L}$ ). In the FAM-only cycles (arm 2), the participants proceeded with the progesterone test as soon as the infertile phase occurred after ovulation, as defined by the FAM being used. The 2 arms were compared with regard to accuracy of identification (yes/ no) of the luteal phase. Statistical analysis to compare the 2 arms consisted of creating a contingency table and applying an exact McNemar test. Participants trying to get pregnant or currently or recently using (6 months) of any type of hormonal contraception were excluded.

\section{Results}

Forty women were assessed for enrollment; 29 fulfilled the criteria and 6 were lost to follow-up (loss of interest, $\mathrm{n}=2$; moved away, $\mathrm{n}=1$; medical reasons, $\mathrm{n}=3$ ). The 23 women enrolled had the following characteristics: age range 20 to 48 years; 
Table 1. Absolute and Relative Proportion of Detection between Luteinizing Hormone (LH) Adjunct and Fertility Awareness Methods (FAMs)

\begin{tabular}{lccccc}
\hline Method & Detected Cycles & No. & Proportion & Relative Risk of FAM/LH (95\% CI) & Absolute Difference (LH - FAM) \\
\hline FAM & 20 & 23 & 0.87 & $0.87(0.72-0.97)$ & $0.13(0.03-0.28)$ \\
LH kit/FAM & 23 & 23 & 1.00 & & \\
\hline
\end{tabular}

mostly white $(\mathrm{n}=18)$; using mucus-based method $(\mathrm{n}=14)$ or mucus temperature $(\mathrm{n}=9)$; all Canadian; and all with a high school education or higher.

Each woman supplied information on 2 cycles, for a total of 46 cycles. Mean cycle length was 29.3 days. For the FAM cycles, mean length was 29 days. For the cycles using LH kits, mean length was 29.5 days. There was no significant difference between cycle lengths for each woman. All the women correctly identified the luteal phase using the LH kit as an adjunct. Three phases were not correctly identified using FAM only, giving a 0.13 absolute difference (Table 1). A mean of 15 kits were used per cycle.

\section{Discussion}

In this pilot study, we found that the use of urinary LH kits with a sensitivity of $20 \mathrm{mIU} / \mathrm{mL}$ may provide a useful adjunct for those women with difficulty identifying their postovulation period. This study did not, however, attempt to develop a new FAM method but to explore an option for those women who need to gain extra confidence. There are a few limitations to our study. LH kits may not provide a $100 \%$ confirmation in clinical circumstances outside our population of study given the variability in the $\mathrm{LH}$ secretion during the cycle $^{3}$; such scenarios may include, for instance, breastfeeding, very long anovulatory cycles, ex- tremely short LH surges, and premenopause. In addition, this study did not address the beginning of the fertile phase, which still requires the use of a reliable first indicator for those women who do not want to conceive. Theoretically, the above-mentioned problems could be easily addressed with the development of simpler and cheaper urinary tests or monitors that use the metabolites of estrogen and progesterone. ${ }^{4}$ Future promising research could involve the use of several urinary metabolites (estradiol, LH, progesterone) $)^{2}$ with or without the combination of clinical signs such as cervical mucus to ascertain both the fertile and infertile phases of the menstrual cycle. This pilot study could be used as an example for future research.

\section{References}

1. Manhart MD, Duane M, Lind A, et al. Fertility awareness-based methods of family planning: a review of effectiveness for avoiding pregnancy using SORT. Osteopath Fam Physician 2013;5:2-8.

2. Ecochard R, Leiva R, Bouchard T, et al. Use of urinary pregnanediol 3-glucuronide to confirm ovulation. Steroids 2013;78:1035-40.

3. Direito A, Bailly S, Mariani A, Ecochard R. Relationships between the luteinizing hormone surge and other characteristics of the menstrual cycle in normally ovulating women. Fertil Steril 2013;99: 279-85.

4. Djerassi C. Fertility awareness: jet-age rhythm method? Science 1990;248:1061-2. 